\title{
5.1. INFORMATION OBTAINABLE FROM LASER RANGE MEASUREMENTS TO A LUNAR CORNER REFLECTOR
}

\author{
C. O. Alley \\ (Dept. of Physics and Astronomy, University of Maryland, \\ College Park, Md., U.S.A.) \\ and
}

\author{
P. L. BENDER \\ (Joint Institute for Laboratory Astrophysics, University of Colorado and National \\ Bureau of Standards, Boulder, Colo., U.S.A.)
}

\begin{abstract}
It has been proposed to the U.S. National Aeronautics and Space Administration that optical retro-reflector packages be placed on the lunar surface under either the Surveyor or Apollo Programs. Methods for measuring the range to the reflectors with an expected accuracy of $15 \mathrm{~cm}$ have been presented. The new technique is briefly discussed, and an analysis of the determination of geocentric longitude is given, indicating a potential uncertainty of $0.25 \times 10^{-3} \mathrm{~s}$ of time.

\section{RÉSUMÉ}

Il a été proposé à l'Administration nationale américaine de l'aéronautique et de l'espace (NASA) de poser sur la surface de la Lune un réflecteur artificiel de lumière - cataphote - dans le cadre de l'un de ses programmes - "Surveyor" ou "Apollo". Diverses méthodes ont été présentées, assurant aux mesures de la distance aux réflecteurs une précision de $15 \mathrm{~cm}$. La technique nouvelle est discutée succintement, et une analyse est présentée de la méthode de détermination de la longitude géocentrique avec une précision de $0.25 \times 10^{-3} \mathrm{~s}$ horaires.
\end{abstract}

\section{Introduction}

In this paper we wish to suggest that precise measurement of the range between stations on the Earth and a bench mark on the Moon consisting of an optical retroreflector, made possible by recent progress in quantum electronics and space capabilities, offers a very useful technique for the study of the topics of this symposium. Increased knowledge of the center of mass motion of the Moon, forced physical librations, and the lunar figure, as well as information on a possible secular variation of the gravitational constant, would also be produced by a successful experiment continued over one or two decades.

A brief description of the method has been published (Alley et al., 1965). A much more detailed analysis of the science and technology has been made in a formal 
proposal to carry out the experiment, submitted to the National Aeronautics and Space Administration of the United States by a group* consisting of the present authors (who are representing the group) and D. Brouwer (Yale University, now deceased), D. G. Currie (University of Maryland), R. H. Dicke (Princeton University), J.E. Faller (Wesleyan University), G. J.F. MacDonald (University of California at Los Angeles), H.H. Plotkin (Goddard Space Flight Center), S. K. Poultney (University of Maryland), H. Richard (Goddard Space Flight Center), D.T. Wilkinson (Princeton University), U. Van Wijk (University of Maryland, now deceased), and W.M. Kaula (University of California at Los Angeles). Much of this material will be published elsewhere.

There are indications in the Soviet literature of similar plans for the emplacement of a lunar optical corner reflector (Kokurin et al., 1966a, 1966b, 1967).

\section{Technique}

It is well within the capabilities of existing ruby lasers to generate a pulse of radiation of duration $10^{-8} \mathrm{~s}$, with an energy of 10 joules at $6943 \AA$, and an angular radius of $10^{-3} \mathrm{rad}$ from a $2-\mathrm{cm}$ aperture $(\approx 100$ times the diffraction limit). If this beam is recollimated to exit from a good quality $150 \mathrm{~cm}$ aperture, it will have an angular radius of about 2.77 and would illuminate an area on the Moon a few kilometers in extent. The presence of a nearly diffraction limited optical-corner reflector near the center of the pattern (which will be blotchy because of index variations in the atmosphere) will send back a part of the pulse to the receiver without any appreciable spreading in time, as contrasted with the return from the curved lunar surface which will be spread to several microseconds. Also, the return will be many times that of the surface for a modest sized reflector. For a currently considered design, having a mass of several kilograms, the enhancement is two orders of magnitude for an albedo of $10 \%$. Thus, on a single shot it will be possible to measure the round trip time of $\approx 2.5 \mathrm{~s}$ to $10^{-8} \mathrm{~s}$, yielding a range accuracy of $1.5 \mathrm{~m}$. By processing the returns from $\approx 100$ shots, it seems possible to determine the range to $15 \mathrm{~cm}$.

If the transmitting aperture is used also for receiving, there will be a reduction in the signal caused by velocity aberration. For the parameters given above, assuming an enhanced quantum efficiency of $10 \%$, a signal of a few photo-electrons is expected. The number of photo-electrons produced by the background radiation passed by a 3-Å filter during the 10 nanosecond duration of the pulse will be much smaller, even for the sunlit condition on the Moon. Therefore the signal is sufficient for measurement when a range gate is used. A computer program to predict the range to the necessary accuracy at the epoch of firing, which needs to be measured to 0.1 millisecond,

* In June, 1967, NASA endorsed the emplacement of a retro-reflector on the Moon as an official Apollo lunar surface experiment for the earliest possible landing. Responsibility for the design to accomplish the scientific objectives was given to this group. 
is required. With an average power of about 3 watts, corresponding to one shot every $3 \mathrm{~s}$, one could achieve the desired range accuracy in about $5 \mathrm{~min}$.

It is expected that continued progress in laser technology will lead to smaller beam divergences and to shorter pulse lengths than have been used in the above analysis. Ground stations with smaller apertures and less complex electronic processing equipment will then be possible.

It should be emphasized, as is explained in more detail below, that the ranging technique is independent of:

(1) angular errors caused by the atmospheric index of refraction,

(2) knowledge of the local vertical direction.

\section{Range Method for Measuring Geocentric Longitudes}

In this paper we will limit ourselves mainly to the determination of differences in geocentric longitude between stations. Let $t_{M}$ be the time at which the distance from an observing station to the reflector is minimum. We assume that measurements $U_{1}$ and $U_{2}$ of the distance are made at times $t_{1}$ and $t_{2}$ which are respectively about 4 hours before and 4 hours after $t_{M}$ (see Figure 1). For simplicity, the motion of the Moon is neglected. If the difference in the observed distances is small, we find the following formula for $t_{M}$ :

$$
t_{\mathrm{M}}=\frac{1}{2}\left(t_{1}+t_{2}\right)+\frac{U_{1}-U_{2}}{2 \omega R \sin \phi} .
$$

Here $\omega$ is the rotation rate of the earth, $R$ is the distance of the station from the axis of rotation, and $2 \phi$ is the angle of rotation between $t_{1}$ and $t_{2}$. For $R=5 \times 10^{6} \mathrm{~m}$ and an uncertainty of $15 \mathrm{~cm}$ in $U_{1}-U_{2}$, the resulting uncertainty in $t_{M}$ would be 0.25 millisec. From similar measurements at two stations the difference in geocentric longitude would be obtained.

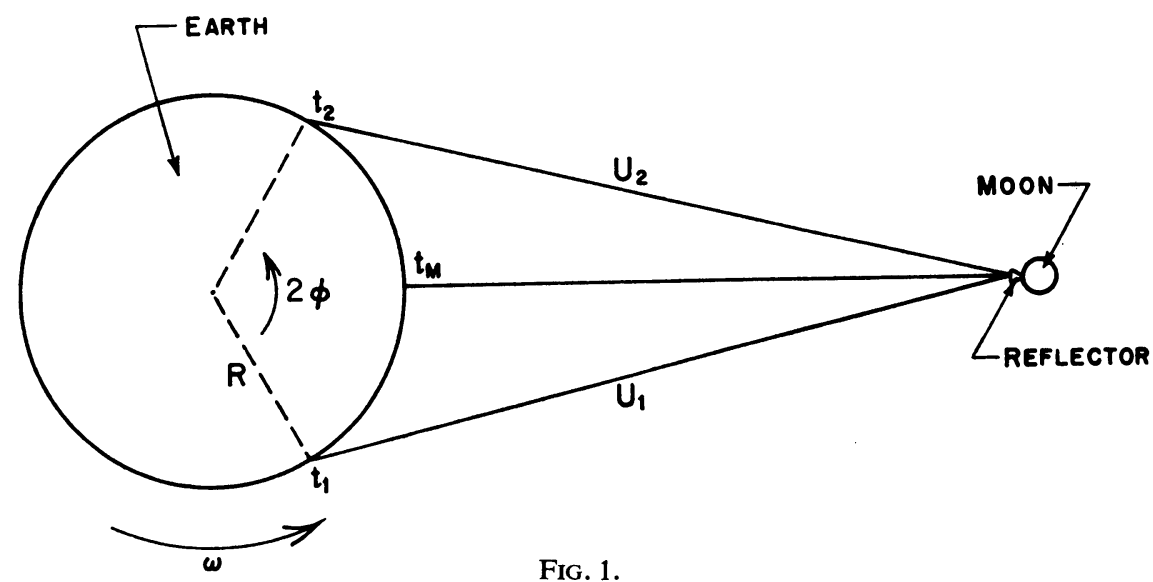




\section{Accuracy of $U_{1}-U_{2}$}

Random Errors: For 10 nanosecond long laser pulses, about 100 pulses would be required to reduce the random error due to the pulse length to $15 \mathrm{~cm}$. Since the use of a larger number of pulses per day appears practical and since information on longitude differences can be accumulated over extended periods, it is probable that the limitation will come from systematic errors.

Time Delays: We have estimated that the absolute calibration of time delays in the photo-multiplier and electronics can be done to somewhat less than one nanosecond, but in this application it is the difference in the time delays between the measurement of $U_{1}$ and $U_{2}$ which is important. While variation in the time delay over the eight hour interval between the measurements will probably be observed, there is no apparent reason for a systematic difference.

Earth Tides: The contribution from the earth tides would be expected to be nearly the same for the two measurements if the station is not too near the ocean.

Lunar Motion: After six months to a year of lunar distance measurements, it will be possible to obtain a good fit for the most important parameters in the Hill-BrownEckert theory of the lunar motion and for the lunar librations and location of the reflector with respect to the lunar center of mass. This will make it possible to predict the change over any period of eight hours in the distance from the center of the Earth to the reflector on the Moon with an accuracy of about $2 \mathrm{~cm}$.

Atmospheric Index of Refraction: Range uncertainties due to this source have been studied extensively for microwaves (Bean and Thayer, 1963). At an elevation angle of $20 \mathrm{deg}$ above the horizon, bending of the path contributes negligible error and the total extra optical path length due to the atmosphere is about $6 \mathrm{~m}$. For light this additional path can be predicted to about $6 \mathrm{~cm}$, which is a factor 2 better than for microwaves because of the much lower sensitivity to water vapor (G.D. Thayer, private communication). The difference between the atmospheric errors in $U_{1}$ and $U_{2}$ will not necessarily be smaller because of diurnal effects and time variations, but it should still be substantially reduced when the observed difference in longitude between two stations is averaged over a month.

It is worthwhile to consider the effect of an east-west gradient in the index of refraction across the station of $1 \times 10^{-8}$ per $\mathrm{km}$. This corresponds to a temperature gradient of $0.01^{\circ} \mathrm{C} / \mathrm{km}$ if the pressure is constant. We assume for simplicity that the atmosphere is uniform in density up to $10 \mathrm{~km}$ and zero above that height. For vertical observations, the above gradient leads to a deflection of 0.02 (of arc), or about a $60 \mathrm{~cm}$ error in the station position observed with a photographic zenith tube. The corresponding difference in the distances measured through the atmosphere at angles of $+60^{\circ}$ and $-60^{\circ}$ from the zenith due to this gradient is $0.2 \mathrm{~cm}$. While the two distance measurements are actually made at times eight hours apart, the above example still gives reason to expect the mean value of the atmospheric error over extended 
periods to be much less for methods based on distance measurements than for those based on angle measurements.

\section{Continental Drift and Polar Motion}

The above discussion indicates that the systematic and random errors expected in determining the difference in geocentric longitude of two stations may be small. However, observations from a number of stations would be necessary in order to separate the effects of polar motion from changes in longitude due to crustal movements. While the cost of the necessary equipment is high, it seems desirable for the countries which can do so to undertake lunar range measurements if a suitable retroreflector is placed on the Moon.

\section{Other Information}

For the rotation of the Earth with respect to the Moon, some of the above arguments concerning the reduction of errors by averaging do not apply. An estimated accuracy of somewhat better than one millisec of time seems reasonable. It appears that the motion of the Moon with respect to the Sun would be determined with sufficient accuracy so that effectively the rotation of the Earth with respect to the Sun would be obtained. Changes in the difference in latitude of two stations could be measured with an accuracy of about 0.01 (of arc). The distance of the observing station from the axis of rotation would be determined to about $30 \mathrm{~cm}$ and the distance above the equatorial plane to about $2 \mathrm{~m}$. For stations at nearly the same latitude but about $120^{\circ}$ different longitude, the distance between the stations would be determined to about $50 \mathrm{~cm}$ (relative longitude to about $15 \mathrm{~cm}$ ).

\section{References}

Alley, C. O., Bender, P. L., Dicke, R. H., Faller, J. E., Franken, P. A., Plotkin, H. J., Wilkinson, D.T. (1965) Optical Radar Using a Corner Reflector on the Moon, J. geophys. Res., 70, 2267.

Bean, B.R., Thayer, G.D. (1963) Comparison of Observed Atmospheric Radar Refraction Effects with Values Predicted Through the Use of Surface Weather Observation, J. Res. NBS, 67D, 273.

Kokurin, Yu. L., Kurbasov, V. V., Lobanov, V. F., Mozhzherin, V. M., Sukhanovskiy, A. N., Chernyk, N.S. (1966a) Measurement of the Distance to the Moon by Optical Radar, JETP Letters, 3, 139.

Kokurin, Yu. L., Kurbasov, V. V., Lobanov, V. F., Mozhzherin, V. M., Sukhanovskiy, A. N., Chernyk, N.S. (1966b) On the Possibility of Measuring the Shape and Orbit Parameters of the Moon by Optical Location, Kosmicheskiye Issledovaniya, 4, 414-426.

Kokurin, Yu. L., Kurbasov, V. V., Lobanov, V. F., Mozhzherin, V. M., Sukhanovskiy, A. N., Chernyk, N.S. (1967) On the Possibility of Refining by Means of Optical Location Some Astronomical Parameters of the System Earth-Moon, Kosmicheskiye Issledovaniya, 5, 219-224. 